\title{
Complete genetic linkage maps from an interspecific cross between Fusarium circinatum and Fusarium subglutinans
}

\author{
L. De Vos ${ }^{a, b}$, A.A. Myburg ${ }^{a, b}$, M.J. Wingfield ${ }^{b}$, A.E. Desjardins ${ }^{c}$, T.R. Gordon ${ }^{d}$ and \\ B.D. Wingfield ${ }^{\mathrm{a}, \mathrm{b}}$
}

${ }^{\mathrm{a}}$ Department of Genetics, Faculty of Natural and Agricultural Sciences, University of Pretoria, Pretoria 0001, South Africa

${ }^{b}$ Forestry and Agricultural Biotechnology Institute (FABI), University of Pretoria, Pretoria 0001, South Africa

${ }^{\mathrm{N}}$ National Centre for Agricultural Utilization Research, United States Department of Agriculture, 1815 N. University Street, Preoria, IL 61604, USA

${ }^{\mathrm{d}}$ Department of Plant Pathology, University of California, Davis, CA 95616, USA

\section{Abstract}

The Gibberella fujikuroi complex includes many plant pathogens of agricultural crops and trees, all of which have anamorphs assigned to the genus Fusarium. In this study, an interspecific hybrid cross between Gibberella circinata and Gibberella subglutinans was used to compile a genetic linkage map. A framework map was constructed using a total of 578 AFLP markers together with the mating type (MAT-1 and MAT-2) genes and the histone (H3) gene. Twelve major linkage groups were identified $(n=12)$. Fifty percent of the markers showed significant deviation from the expected 1:1 transmission ratio in a haploid $\mathrm{F}_{1}$ cross $(P<0.05)$. The transmission of the markers on the linkage map was biased towards alleles of the G. subglutinans parent, with an estimated $60 \%$ of the genome of $F_{1}$ individuals contributed by this parent. This map will serve as a powerful 
tool to study the genetic architecture of interspecific differentiation and pathogenicity in the two parental genomes.

\section{Article Outline}

1. Introduction

2. Materials and methods

2.1. Fungal isolates

2.2. DNA isolation

2.3. AFLP analysis

2.4. Additional marker analysis

2.5. Framework linkage map construction

2.6. Bin mapping of accessory markers

2.7. Estimated genome coverage and length

3. Results

3.1. DNA isolation and AFLP analysis

3.2. Framework linkage map construction

3.3. Bin mapping of accessory markers

3.4. Estimated genome coverage and length

4. Discussion

Acknowledgements

References

\section{Introduction}

Gibberella fujikuroi (Sawada) Wollenw. is the species complex associated with species that have anamorphs in Fusarium section Liseola. These include many important fungal pathogens of agricultural crops and trees. The Fusarium species associated with this complex include at least eleven different biological species (mating population $\mathrm{A}-\mathrm{K}$ ), 
which are reproductively isolated ([Nirenberg and O'Donnell, 1998], [Samuels et al., 2001], [Zeller et al., 2003], [Phan et al., 2004] and [Lepoint et al., 2005]).

Traditionally, the morphological species concept has been used to describe species in the G. fujikuroi complex, but differentiation between species following this approach has generally been unsatisfactory. One example is Fusarium subglutinans sensu lato within which three mating populations $(\mathrm{B}, \mathrm{E}$ and $\mathrm{H})$ have been identified, based on patterns of inter-isolate fertility. Recognition of these mating populations as distinct species is supported by multigene phylogenies ([Nirenberg and O'Donnell, 1998] and [O'Donnell et al., 1998]). Fusarium subglutinans sensu stricto is used for strains isolated from maize and belongs to mating population E ([Nirenberg and O'Donnell, 1998] and [O'Donnell et al., 1998]). Fusarium circinatum is the name applied to isolates from pine that cause pitch canker, which are associated with mating population $\mathrm{H}$ of the G. fujikuori complex ([Nirenberg and O’Donnell, 1998] and [Britz et al., 1999]). The biological species concept has been used extensively to characterise other species in the G. fujikuroi complex. However, this approach has limited value because the majority of species have no apparent sexual stage (Steenkamp et al., 2002).

Fusarium circinatum (mating population $\mathrm{H}$ ), also known as the pitch canker fungus, is a pathogen of many pine species, and is especially damaging to Pinus patula and Pinus radiata ([McCain et al., 1987] and [Viljoen and Wingfield, 1994]). This fungus was first discovered in the United States in 1946 (Hepting and Roth, 1946) and has since spread to many other parts of the world including South Africa. In the latter country it causes root rot and damping off of $P$. patula and other susceptible pine species in seedling nurseries (Viljoen and Wingfield, 1994).

Fusarium subglutinans (mating population E) is a common pathogen of domesticated maize (Zea mays spp. mays). Desjardins et al. (2000) studied isolates of Fusarium from maize and the closely related wild teosinte (Zea spp.) in Mexico and Central America, in an attempt to characterize these isolates and determine an appropriate mating population for them. Strains identified based on morphology as F. subglutinans from maize and teosinte, were infertile when crossed with tester strains from mating populations $\mathrm{B}, \mathrm{E}$ and H. However, one strain of $F$. subglutinans isolated from teosinte was moderately fertile in a cross with an isolate of $F$. circinatum (mating population H). Desjardins et al. (2000) 
suggested that all $F$. subglutinans strains treated in this study represent a fourth distinct mating population associated with $F$. subglutinans. This was due to the almost complete infertility with the standard tester strains. Steenkamp et al. (2001) applied phylogenetic analyses and sexual compatibility tests to show that $F$. subglutinans isolates from teosinte belong to mating population E. They are phylogenetically more closely related to each other than to other mating populations within the G. fujikuroi species complex ([O’Donnell et al., 1998], [Steenkamp et al., 1999] and [Steenkamp et al., 2000]), indicating they share a greater ancestry. Hybridization of closely related fungal species, such as mating population $\mathrm{E}$ and $\mathrm{H}$ forming the basis of this study, has been documented in other fungi (Lind et al., 2005) as well as within the Liseola section of Fusarium ([Desjardins et al., 1997] and [Leslie et al., 2004b]).

Fusarium circinatum and $F$. subglutinans threaten forestry and maize production in South Africa. Both these industries are considered integral parts of South Africa's economy with maize being considered to be the staple diet of South Africans with approximately $25 \%$ of South Africa's total arable land use being planted to maize. Therefore, both commercial forestry and maize are of economic importance in South Africa and maintaining these sectors is of important to the economy of the country.

The cross between $F$. circinatum and F. subglutinans made by Desjardins et al. (2000) provided us with a unique opportunity to study genetic differentiation using genetic linkage mapping. Genetic linkage maps have also been used to study other fungi including Fusarium verticillioides ([Xu and Leslie, 1996] and [Jurgenson et al., 2002b]) and Fusarium graminearum ([Jurgenson et al., 2002a] and [Gale et al., 2005]). In general, AFLP markers are preferred when generating linkage maps. The objective of our study was to use AFLP markers (Vos et al., 1995) to generate a genetic linkage map for an interspecific cross between Gibberella circinata and Gibberella subglutinans. This map should provide a useful framework for further study of the architecture of the two parental genomes and elucidation of the genetic determinants of pathogenicity to pine. 


\section{Materials and methods}

\subsection{Fungal isolates}

Isolates used for genetic linkage analysis were $\mathrm{F}_{1}$ progeny from the cross between $G$. circinata (maternal parent; MAT-1) and G. subglutinans (paternal parent; MAT-2) from the study of Desjardins et al. (2000). The parents of this cross were isolates MRC7828 (G. subglutinans) and MRC7870 (G. circinata) (Table 1). Ninety-four $\mathrm{F}_{1}$ isolates were randomly selected from 226 viable ascospore progeny obtained from 14 perithecia for use in this study.

Table 1.

Hosts, geographic origins and source of the $\mathrm{F}_{1}$ parents used in this study

\begin{tabular}{|l|l|l|l|}
\hline Isolate $^{\mathbf{a}}$ & Host & Geographic origin & Source \\
\hline MRC7828; Fst51 $^{\mathbf{b}}$ & Zea mays spp. Mexicana & Texcoco, Mexico & A.E. Desjardins \\
\hline MRC7870; Fsp34 & Pinus spp. & California, USA & T.R. Gordon \\
\hline
\end{tabular}

${ }^{a}$ MRC, W.F.O. Marasas, Programme on Mycotoxins and Experimental Carcinogenesis (PROMEC), Medical Research Council, Tygerberg, South Africa.

${ }^{\mathrm{b}}$ F. subglutinans (mating population E).

${ }^{\mathrm{c}}$ F. circinatum (mating population $\mathrm{H}$ ).

\subsection{DNA isolation}

Isolates were grown on half strength PDA (potato dextrose agar; 20\% potato dextrose agar and 5\% agar) for 7 days at $25^{\circ} \mathrm{C}$ in the dark. Mycelium was harvested and $300 \mu \mathrm{l}$ extraction buffer (200 mM Tris [pH 8], $250 \mathrm{mM} \mathrm{NaCl,} 25 \mathrm{mM}$ EDTA [pH 8], 0.5\% w/v SDS) was added (Raeder and Broda, 1985). This mixture was homogenised at $4 \mathrm{~m} / \mathrm{s}$ for 20 s using the Fastprep FP120 (QBIOgene, Farmingdale, NY, USA) system. Following homogenisation, the tissue was frozen in liquid nitrogen and then thawed in boiling water for $5 \mathrm{~min}$. Phenol-chloroform (1:1) extractions were performed $(10,600 \mathrm{~g}$ for $5 \mathrm{~min})$ until all cell debris had been removed. Thereafter, 0.1 volumes of $3 \mathrm{M}$ sodium acetate $(\mathrm{pH} 8)$ and two volumes of cold absolute ethanol were added and the Eppendorf tubes were 
inverted five times. After centrifugation at 10,600 $\mathrm{g}$ for $5 \mathrm{~min}, 1 \mathrm{ml} \mathrm{70 \%}$ ethanol was added to the supernatant and allowed to stand for $5 \mathrm{~min}$ at room temperature (Sambrook et al., 1989). The precipitated DNA was centrifuged for a further $5 \mathrm{~min}$ at $2700 \mathrm{~g}$ and dried under vacuum. The DNA was resuspended in $500 \mu 1$ low TE (10 mM Tris [pH 8], $0.1 \mathrm{mM}$ EDTA).

\subsection{AFLP analysis}

AFLP analysis was performed essentially following the protocol of Vos et al. (1995). Restriction digestion of the genomic DNA was performed using EcoRI and MseI (Zeller et al., 2000). These restriction fragments were ligated to the corresponding enzymespecific oligonucleotide adapters (Vos et al., 1995). Preselective amplifications were performed with zero-base-addition EcoRI and MseI adapter-specific primers using the following PCR conditions: 1 cycle of $30 \mathrm{~s}$ at $72{ }^{\circ} \mathrm{C}, 25$ cycles of $30 \mathrm{~s}$ at $94{ }^{\circ} \mathrm{C}, 30 \mathrm{~s}$ at $56{ }^{\circ} \mathrm{C}$ and $1 \mathrm{~min}$ at $72{ }^{\circ} \mathrm{C}$ with an increase of $1 \mathrm{~s}$ per cycle and a final elongation step of 2 min at $72{ }^{\circ} \mathrm{C}$. Final selective amplifications used EcoRI and MseI primers (Table 2) with two-base-additions. The EcoRI primer was labelled with the infrared dyes, IRDye ${ }^{\mathrm{TM}}$ 700 or IRDye 800 (LI-COR, Lincoln, NE). PCR conditions were as follows: 13 cycles of $10 \mathrm{~s}$ at $94{ }^{\circ} \mathrm{C}, 30 \mathrm{~s}$ at $65^{\circ} \mathrm{C}$ with a decrease of $0.7^{\circ} \mathrm{C}$ per cycle and $1 \mathrm{~min}$ at $72{ }^{\circ} \mathrm{C}$ followed by 23 cycles of $10 \mathrm{~s}$ at $94{ }^{\circ} \mathrm{C}, 30 \mathrm{~s}$ at $56^{\circ} \mathrm{C}$ and $1 \mathrm{~min}$ at $72{ }^{\circ} \mathrm{C}$ with an increase of $1 \mathrm{~s}$ per cycle and a final elongation step of $1 \mathrm{~min}$ at $72{ }^{\circ} \mathrm{C}$.

Table 2.

AFLP primer combinations used in this study

\begin{tabular}{|l|l|l|}
\hline Primer combination & No. polymorphisms & \% Polymorphic bands/primer \\
\hline M-AA + E-AA (700) & 61 & 62.9 \\
\hline M-AA + E-CC (700) & 44 & 56.4 \\
\hline M-AA + E-AC (700) & 54 & 68.4 \\
\hline M-AA + E-TC (800) & 55 & 70.7 \\
\hline
\end{tabular}




\begin{tabular}{|l|l|l|}
\hline Primer combination & No. polymorphisms & \% Polymorphic bands/primer \\
\hline M-GA + E-CC (700) & 32 & 48.5 \\
\hline M-GA + E-AC (700) & 40 & 62.5 \\
\hline M-GA + E-TC (800) & 42 & 56.0 \\
\hline M-AC + E-AA (700) & 26 & 26.5 \\
\hline M-AG + E-AC (700) & 32 & 53.3 \\
\hline M-AT + E-AC (700) & 45 & 70.3 \\
\hline M-CA + E-TC (800) & 63 & 66.3 \\
\hline M-AA + E-TT (800) & 45 & 48.9 \\
\hline M-AG + E-AA (700) & 39 & 42.4 \\
\hline Total & 578 & \\
\hline Average & $44.5 \pm 11.3$ & 56.4 \\
\hline
\end{tabular}

In the first column, the primer combinations are given for the $M s e I(\mathrm{M})$ selective nucleotides and the $E c o$ RI (E) selective nucleotides. The value given in parenthesis refers to the IRDye ${ }^{\mathrm{TM}}$ (LI-COR, Lincoln, NE) used for fragment analysis.

${ }^{a}$ Only the markers used for framework map construction. AFLP fragment analysis was performed on a model 4200 LI-COR ${ }^{\circledR}$ automated DNA sequencer as described by Myburg et al. (2001). Electrophoresis run parameters were set to the following: $1500 \mathrm{~V}, 35 \mathrm{~mA}, 35 \mathrm{~W}, 45^{\circ} \mathrm{C}$, motor speed 3 and signal filter 3 . Electrophoresis prerun time was set to $30 \mathrm{~min}$ and the run time to $4 \mathrm{~h}$.

Digital gel images obtained from the LI-COR system were analysed using the Saga ${ }^{\mathrm{MX}}$ AFLP ${ }^{\circledR}$ Analysis Software package (LI-COR) according to the manufacturers' instructions. Only markers that were polymorphic for the two hybrid parent strains were 
scored with a ' 0 ' indicating absence, ' 1 ' indicating presence of bands and ' $\mathrm{X}$ ' indicating missing data.

\subsection{Additional marker analysis}

PCR identification of the mating types of all the isolates was performed as described by Steenkamp et al. (2000). It was not possible to perform the multiplex PCR as described, but superior results were achieved when the $M A T-1$ or $M A T-2$ primer sets were used separately. An annealing temperature of $65^{\circ} \mathrm{C}$ was used for the $M A T-2 \mathrm{PCR}$ and $67{ }^{\circ} \mathrm{C}$ for $M A T-1$.

Because the $\mathrm{F}_{1}$ isolates were hybrids of $F$. circinatum and $F$. subglutinans, PCR-RFLP analysis of the histone $\mathrm{H} 3$ gene was used to distinguish between parental alleles (Steenkamp et al., 1999). This PCR-RFLP technique successfully determined the parental origin of the histone $\mathrm{H} 3$ alleles segregating in the $\mathrm{F}_{1}$ isolates. The results of all these amplifications were scored as a ' 0 ' for band absent and ' 1 ' for band present.

\subsection{Framework linkage map construction}

$\chi^{2}$ analysis was performed on all markers to test for departure from the expected Mendelian segregation ratio (1:1) using a significance threshold of $\alpha=0.05$. All markers, including those showing transmission ratio distortion, were included in framework map construction in order to optimise map coverage.

Based on the origin of each marker, the data were separated into two parental data sets. Linkage analysis was performed on the separate and joint data sets to obtain separate parental framework linkage maps and a $F_{1}$ framework linkage map. A maternal map of the $F$. circinatum parent and a paternal map of the $F$. subglutinans parent were generated, representing the two linkage phases of the $F_{1}$ map. The parental (linkage phase) maps were constructed separately to increase the confidence of marker ordering in the $F_{1}$ map. Markers having greater than $10 \%$ missing data were dropped from the data sets before framework map construction in MAPMAKER Macintosh V2.0 (Lander et al., 1987). Data were regarded as $\mathrm{F}_{2}$ backcross configuration to accurately analyse segregation in the haploid $\mathrm{F}_{1}$ genomes (Xu and Leslie, 1996). The Kosambi mapping function was used. The haploid chromosome number of $F$. subglutinans is known $(n=12)(\mathrm{Xu}$ et al., 1995). 
This information was used when distributing markers into linkage groups by evaluating the LOD linkage thresholds from 6 to 14, in incremental steps of 1.0, by using the 'Group' command (Myburg et al., 2003). The parental marker sets was separated into at least 12 linkage groups at LOD thresholds of 9 and 10.

To select framework markers, markers in each linkage group were subjected to the 'First Order' command of MAPMAKER to attain a starting order. Using the 'Drop Marker' command, internal markers that expanded the map by more than $11 \mathrm{cM}$ were dropped. After a marker had been dropped, the 'First Order' step was repeated. Using the 'Ripple' function, the support of the remaining markers was evaluated. Markers that did not have a LOD interval support of at least 1.5 were removed from the map. The 'First Order' step was again repeated after each marker was dropped. Finally the terminal markers were evaluated with the 'TwoPoint/LOD Table' command. Terminal markers that showed stronger pairwise linkage to internal markers than to adjacent markers were dropped (Myburg et al., 2003).

In order to combine the parental maps and construct a single integrated map (referred to herein as the " $F_{1}$ map"), marker presence/absence data from the maternal data set was recoded to indicate that band absent (' 0 ') represented $F$. circinatum markers. The two data sets (the recoded maternal data set and the paternal data set) were then combined into one data set and map construction was performed using the MAPMAKER program as described above. Markers were distributed into linkage groups using the 'Group' command by evaluating the LOD linkage thresholds from 6 to 14, in incremental steps of 1.0. The mapping set was separated into 12 major linkage groups at a LOD threshold of 9. Higher thresholds were used for the 'Drop Marker' command and 'Ripple' functions, with markers expanding the map length by $10 \mathrm{cM}$ and not having a LOD interval support of at least 3.0 being dropped respectively.

Data from the $F_{1}$ map were subjected to the Graphical GenoTyping program (GGT) (Van Berloo, 1999). This was used to inspect the distribution of crossovers for each chromosome. This program was also used to determine if any nonrecombinant or duplicate progeny was found to show that this interspecific cross was the product of a heterothallic event. 


\subsection{Bin mapping of accessory markers}

AFLP markers that did not meet framework marker criteria were mapped to the framework map as accessory markers using the bin mapping function of the MapPop V1.0 program. This program places accessory markers into map intervals contained in a previously constructed high confidence framework map (Vision et al., 2000). Only markers with $P>0.95$ were placed into framework intervals. MapPop does not allow for the placement of accessory markers outside the terminal framework markers of each linkage group. Thus, AFLP markers not placed with MapPop were assessed for linkage to terminal markers using the 'Two Point/LOD' function of MAPMAKER (Myburg et al., 2003). Markers showing any linkage to the terminal markers at LOD 3.0 were placed as terminal accessory markers.

\subsection{Estimated genome coverage and length}

The total genome length $(L)$ of the $\mathrm{F}_{1}$ map was estimated using the Hulbert estimate (Hulbert et al., 1988) as modified in method 3 of Chakravarti et al. (1991), giving $L$ $=n(n-1) d / k$. Here $n$ is the total number of markers, $d$ is the map distance which corresponds to the LOD threshold at which linkage was determined $(Z)$ and $k$ is the number of markers linked at the LOD threshold of $Z$ or greater. The linkage threshold of LOD 9.0 (Z) was used to estimate genome length as the mapping set was separated into 12 linkage groups at this threshold.

Theoretical map coverage was calculated using the formula $c=1-\mathrm{e}^{-2 d n / L}$, where $\mathrm{c}$ is the proportion of the genome within $d \mathrm{cM}$ of a framework marker, $n$ is the number of framework markers in the map and $L$ is the estimated genome length (Lange and Boehnke, 1982).

\section{Results}

\subsection{DNA isolation and AFLP analysis}

DNA was successfully isolated from the 94 selected $F_{1}$ individuals as well as from the parents of the interspecific cross between $F$. circinatum and $F$. subglutinans. AFLP analyses were performed on these individuals (Table 2). Five hundred and seventy-eight polymorphic AFLP markers were identified with an average of 45 polymorphisms per 
primer combination. In total, $56 \%$ of AFLP fragments were polymorphic. One marker was dropped for linkage analysis as it had more than $10 \%$ missing data. Missing data was defined as bands that could not be scored with confidence due to local gel irregularities, weak amplification, etc. This represented only $1 \%$ of the final data set.

Five hundred and eighty-two markers (578 AFLP markers and four other genetic markers) were generated. The parent-specific alleles of the MAT and H3 marker loci were considered as four different markers for mapping purposes. Of the 582 markers, $50 \%$ deviated significantly from the expected $1: 1$ ratio for a haploid $F_{1}$ cross $(\alpha=0.05$, Table 3). One hundred and seventy-nine markers (31\%) differed at the $1 \%$ level of significance and $97(17 \%)$ at the $0.1 \%$ level of significance (results not shown). Only 12 of the markers that showed transmission ratio distortion at $\alpha=0.05$ were skewed towards the $F$. circinatum parent with the remainder being skewed towards the $F$. subglutinans parent. The number of markers generated from each parent (296 from F. subglutinans and 286 from $F$. circinatum $)$ did not differ significantly $(P=0.68)$.

Table 3.

Summary of markers in the framework linkage maps

\begin{tabular}{|c|c|c|c|}
\hline & $\begin{array}{l}\text { F. subglutinans } \\
\text { (paternal) }\end{array}$ & $\begin{array}{l}\text { F. circinatum } \\
\text { (maternal) }\end{array}$ & $\begin{array}{l}\text { F }_{1} \text { hybrid } \\
\text { (combined) }\end{array}$ \\
\hline \multicolumn{4}{|l|}{ Markers } \\
\hline Total no. of markers & 296 & 286 & 582 \\
\hline $\begin{array}{l}\text { No. of markers showing } \\
\text { transmission ratio distortion }^{\text {a }}\end{array}$ & $128(43.2 \%)$ & $164(57.3 \%)$ & $292(50.2 \%)$ \\
\hline $\begin{array}{l}\text { Markers included in } \\
\text { framework map }\end{array}$ & $104(35.1 \%)$ & $148(51.7 \%)$ & $252(43.3 \%)$ \\
\hline $\begin{array}{l}\text { No. of markers in framework } \\
\text { map showing disortion }^{\mathbf{a}}\end{array}$ & $54(51.9 \%)$ & $85(57.4 \%)$ & $139(55.2 \%)$ \\
\hline
\end{tabular}




\begin{tabular}{|c|c|c|c|}
\hline & $\begin{array}{l}\text { F. subglutinans } \\
\text { (paternal) }\end{array}$ & $\begin{array}{l}\text { F. circinatum } \\
\text { (maternal) }\end{array}$ & $\begin{array}{l}\text { F }_{1} \text { hybrid } \\
\text { (combined) }\end{array}$ \\
\hline No. of accessory markers & $159(53.7 \%)$ & $112(39.2 \%)$ & $271(46.6 \%)$ \\
\hline No. of markers not mapped & $33(11.1 \%)$ & $26(9.1 \%)$ & $59(10.1 \%)$ \\
\hline \multicolumn{4}{|l|}{ Framework maps ${ }^{\mathbf{c}}$} \\
\hline \multicolumn{4}{|l|}{12 linkage groups each } \\
\hline $\begin{array}{l}\text { Average linkage group size } \\
(\mathrm{cM})\end{array}$ & 138.7 & 131.3 & 231.2 \\
\hline $\begin{array}{l}\text { Average framework marker } \\
\text { spacing }(\mathrm{cM})^{\mathbf{d}}\end{array}$ & 18.1 & 11.6 & 11.6 \\
\hline Observed map length $(\mathrm{cM})^{\mathbf{e}}$ & 1664.4 & 1575.5 & 2774.4 \\
\hline $\begin{array}{l}\text { Physical distance per unit of } \\
\text { recombination }{ }^{\mathbf{f}}(\mathrm{kb} / \mathrm{cM})\end{array}$ & 32.5 & 34.4 & 19.5 \\
\hline \multicolumn{4}{|l|}{ Estimation of genome length } \\
\hline $\begin{array}{l}\text { Hulbert estimate of genome } \\
\text { length }(\mathrm{cM})\end{array}$ & & & 2331.7 \\
\hline \multicolumn{4}{|l|}{ Framework map coverage ${ }^{\mathbf{g}}$} \\
\hline $\begin{array}{l}\text { Map coverage }(c \times 100 \%) \text { at } \\
d=20 \mathrm{cM}\end{array}$ & & & $98.7 \%$ \\
\hline
\end{tabular}




\begin{tabular}{|l|l|l|l|}
\hline & $\begin{array}{l}\text { F. subglutinans } \\
\text { (paternal) }\end{array}$ & $\begin{array}{l}\text { F. circinatum } \\
\text { (maternal) }\end{array}$ & $\begin{array}{l}\text { F }_{1} \text { hybrid } \\
\text { (combined) }\end{array}$ \\
\hline $\begin{array}{l}\text { Map coverage }(c \times 100 \%) \text { at } \\
d=10 \mathrm{cM}\end{array}$ & & $88.5 \%$ \\
\hline
\end{tabular}

a $5 \%$ level of significance $(\alpha)$ used to determine the departure of markers from the expected ratio of 1:1 of a haploid cross.

${ }^{\mathrm{b}}$ AFLP markers that were not placed in the framework maps were mapped to the framework map using the bin mapping function of MapPop. Terminal markers were placed using the 'Two Point/LOD' function of MAPMAKER.

${ }^{\mathrm{c}}$ Distances are in centiMorgan (cM) Kosambi.

${ }^{\mathrm{d}}$ Calculated by dividing the summed length of all the linkage groups by the number of framework marker intervals (number of framework markers minus the number of linkage groups).

${ }^{\mathrm{e}}$ Based on the classical estimate of recombination (r).

${ }^{\mathrm{f}}$ Xu et al. (1995) estimated the genome size of $F$. subglutinans to be $54.1 \mathrm{Mbp}$. This estimate of genome size was used to calculate the physical distance per unit of recombination.

${ }^{\mathrm{g}}$ The Hulbert estimate of genome length was used to estimate the framework map coverage.

\subsection{Framework linkage map construction}

All markers, including those showing transmission ratio distortion, were evaluated during framework map construction using the criteria described before. Using MAPMAKER, mapping sets were separated into at least 12 major linkage groups at LOD thresholds of 9 and 10. Subsequent analyses were performed on the linkage groups obtained at the LOD threshold of 9.0, as at this threshold more markers were incorporated into the 12 major linkage groups.

Twelve linkage groups emerged for the two parental framework maps as well as the $F_{1}$ map (Fig. 1). This corresponds to the haploid chromosome number reported for $F$. subglutinans (Xu et al., 1995). Only 252 markers (43\%) met our criteria for framework 
markers in the $F_{1}$ map (Table 3). Less stringent framework marker criteria were subsequently used for construction of the parental (linkage phase) framework maps to ensure that all markers placed in the $\mathrm{F}_{1}$ map were also present in the parental maps. The 252 markers in the $\mathrm{F}_{1}$ map corresponded to 104 markers in the $F$. subglutinans parental map and 148 markers in the F. circinatum parental map. 
openUP (July 2007)
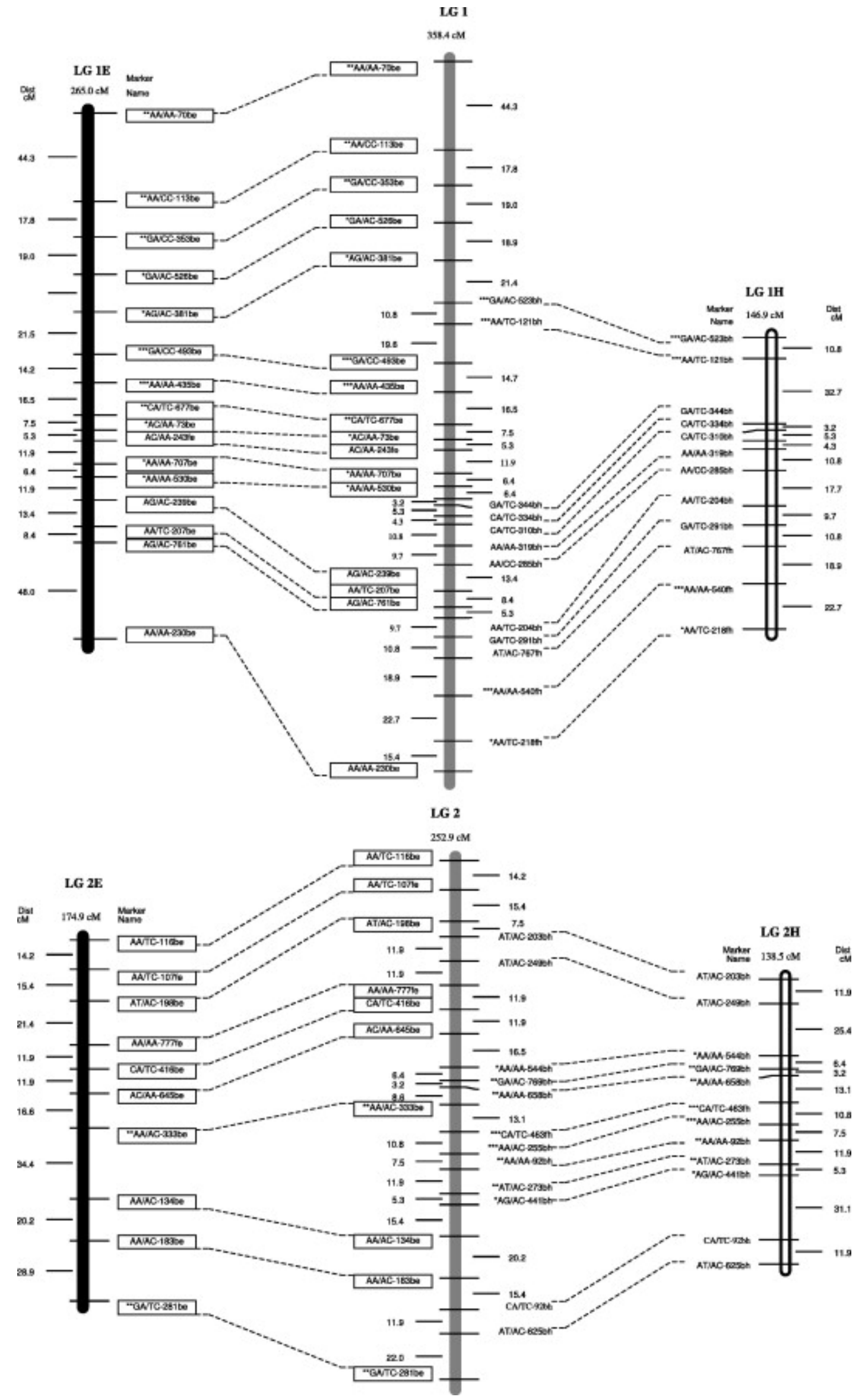
openUP (July 2007)
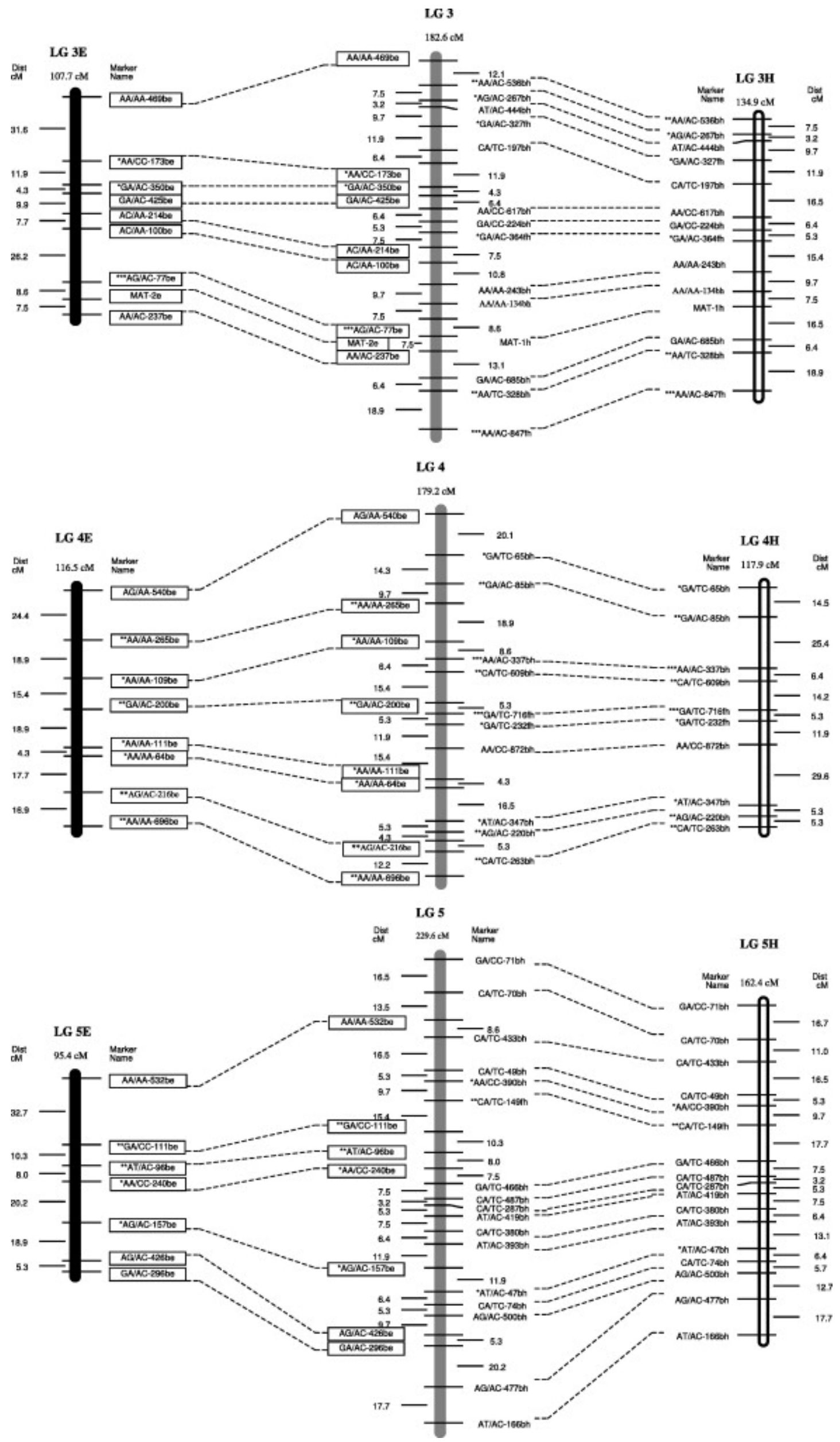

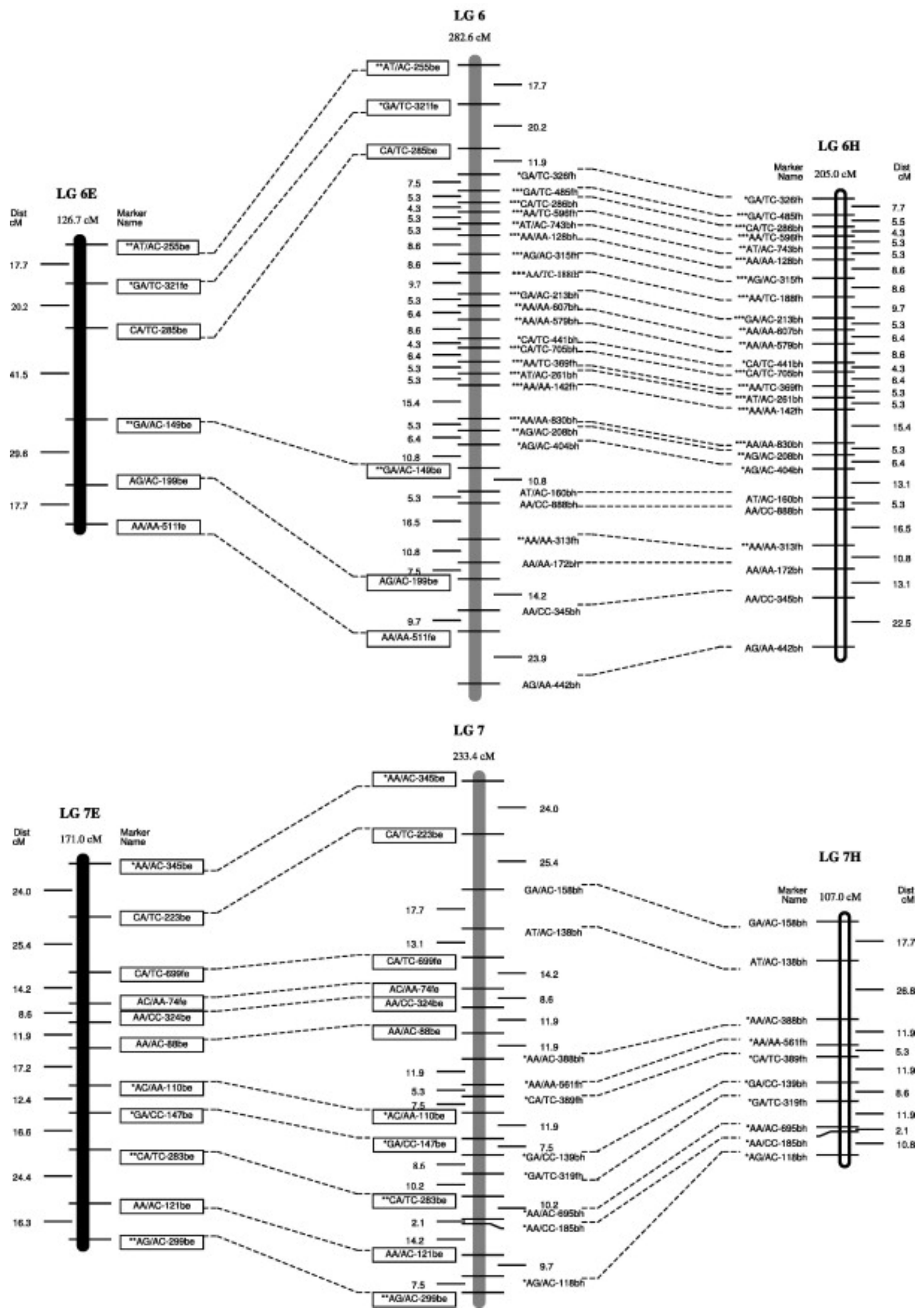


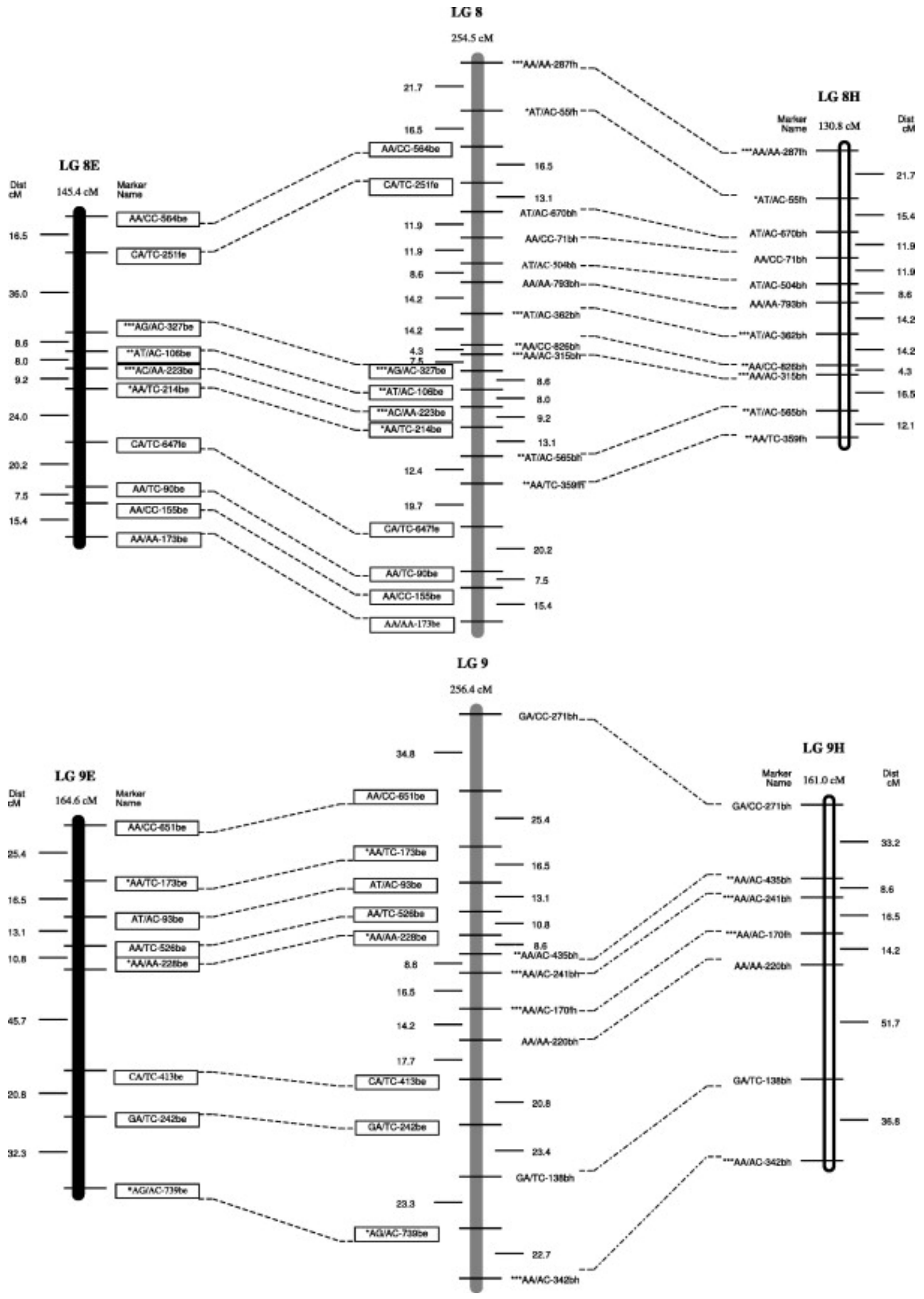


openUP (July 2007)
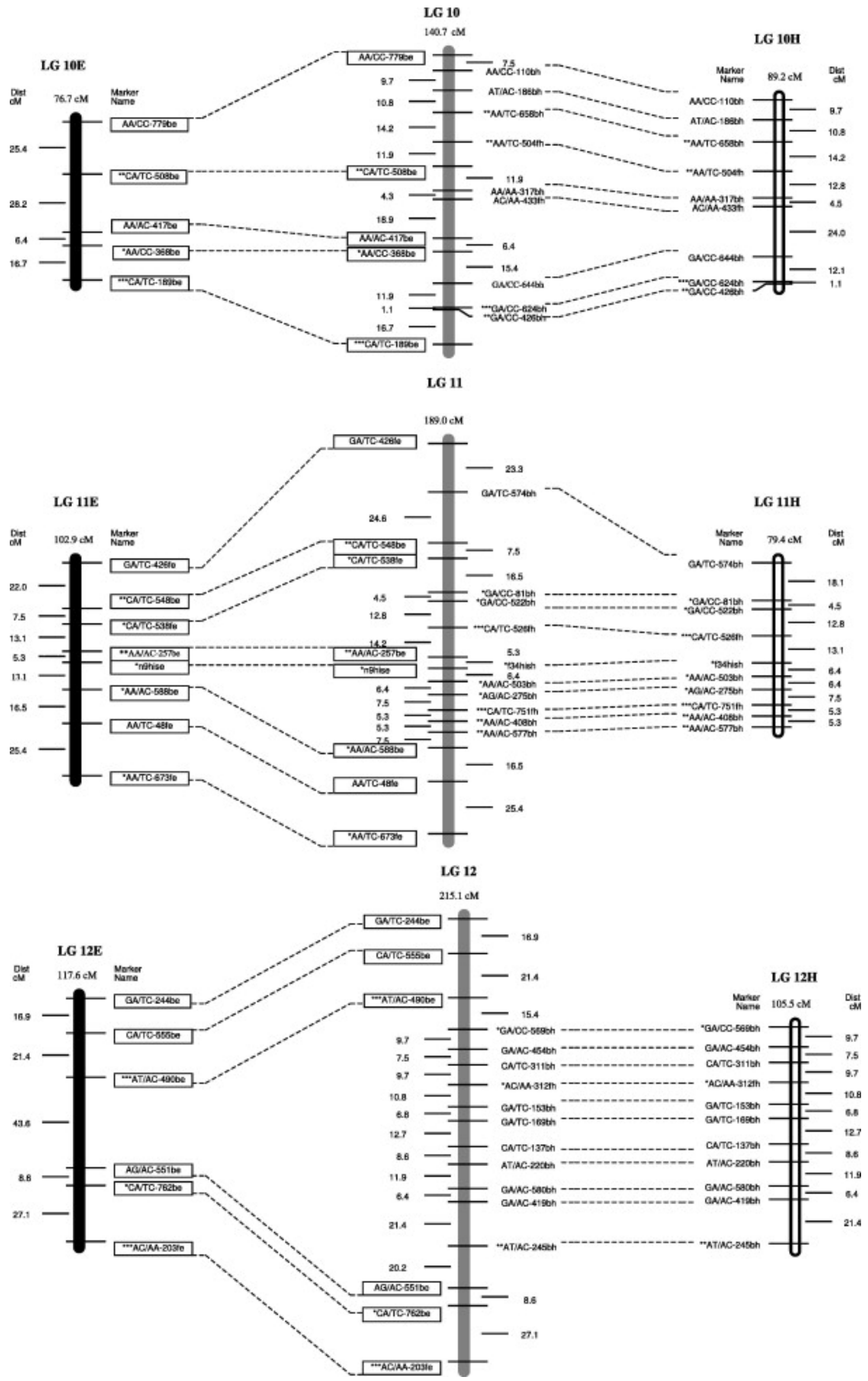
Fig. 1. Integrated framework maps of the cross between $F$. subglutinans and $F$. circinatum. Linkage group numbers followed by an 'E' indicate the $F$. subglutinans parental framework map and a ' $\mathrm{H}$ ' the $F$. circinatum parental framework map. Bars shaded in black designate the $F$. subglutinans linkage map, those that are not shaded the $F$. circinatum linkage map and those that are shaded in grey the integrated F1 map of $F$. subglutinans and F. circinatum. Distances are given in centiMorgan (cM) Kosambi and the total map length of each linkage group is given at the top of each linkage group. Marker names consist of the MseI selective nucleotides followed by the EcoRI selective nucleotides and the molecular size $(\mathrm{bp})$, followed by a $\mathrm{b}$ (bright) or $\mathrm{f}$ (faint) indicating the quality of the fragment, and an 'e' and ' $h$ ' indicating markers originating from either $F$. subglutinans and F. circinatum, respectively. Marker names that are blocked originated from the $F$. subglutinans parent and unblocked from the $F$. circinatum parent. The dotted lines indicate those markers shared between maps. Markers exhibiting transmission ratio distortion are indicated with an asterisk $\left({ }^{*} P<0.05,{ }^{* *} P<0.01,{ }^{* * *} P<.001\right)$. Linkage groups in the $F_{1}$ map ranged in size from $\sim 141 \mathrm{cM}$ (Linkage Group 10) to $\sim$ $358 \mathrm{cM}$ (Linkage Group 1). The total observed length of the map was $2774 \mathrm{cM}$ and the average distance between markers was $12 \mathrm{cM}$. Significantly $(P=0.041)$ more markers from the $F$. circinatum parent were incorporated into the $\mathrm{F}_{1}$ map than from the $F$. subglutinans parent (Table 3).

The MAT idiomorphs mapped to Linkage Group 3 (Fig. 1). Linkage Group 3, therefore, corresponds to chromosome 6 as previously reported for $F$. verticillioides (Xu and Leslie, 1996). The histone H3 gene mapped to linkage group 11 (Fig. 1).

Based on output from the Graphical GenoTyping (GGT) program, the estimated proportion of the genome of the $\mathrm{F}_{1}$ progeny that was descended from $F$. subglutinans was $59.8 \%$ and from $F$. circinatum $39.7 \%$, with $0.5 \%$ of the genome being unknown due to missing data that was scored as ' $\mathrm{X}$ '.

Using the GGT program, the number of progeny lacking any crossovers on each linkage group was determined (Table 4). Of the 12 linkage groups, Linkage Group $5(P<0.05)$ and $6(P<0.001)$ showed significant deviation from the expected 1:1 origin of markers. Both of these linkage groups had a substantial number of markers from $F$. circinatum (Table 4). This was also reflected in the fact that a significantly greater number of 
markers were placed in the $\mathrm{F}_{1}$ map from $F$. circinatum. $\mathrm{F}_{1}$ progeny that received intact linkage groups tended to inherit these from the $F$. subglutinans parent, which was significant for Linkage Group 7 and $11(P<0.05), 1,4,8$ and $12(P<0.01)$ and $10(P$ $<0.001)$. No duplicate progeny was found, supporting the view that the interspecific cross forming the basis of this study was the product of a heterothallic event.

Table 4.

The number of $F_{1}$ individuals with parental types on each linkage group and the origin of framework markers in each linkage group

\begin{tabular}{|l|l|l|l|l|}
\hline Linkage group & \multicolumn{2}{|l|}{ Intact parental linkage group $^{\mathbf{c}}$} & \multicolumn{2}{l|}{ Framework markers } \\
\hline & $\mathbf{H}^{\mathbf{a}}$ & $\mathbf{E}^{\mathbf{b}}$ & $\mathbf{H}^{\mathbf{d}}$ & $\mathbf{E}^{\mathbf{e}}$ \\
\hline 1 & 0 & $10^{* *}$ & 12 & 16 \\
\hline 2 & 6 & 13 & 12 & 10 \\
\hline 3 & 9 & 19 & 14 & 9 \\
\hline 4 & 4 & $17^{* *}$ & 10 & 8 \\
\hline 5 & 10 & 15 & $17^{*}$ & 7 \\
\hline 6 & 6 & 11 & $25^{* * *}$ & 6 \\
\hline 7 & 8 & $18^{*}$ & 10 & 11 \\
\hline 8 & 4 & $19^{* *}$ & 11 & 10 \\
\hline 9 & 4 & 9 & 7 & 8 \\
\hline 10 & 7 & $28^{* * *}$ & 9 & 5 \\
\hline 11 & 10 & $24^{*}$ & 10 & 8 \\
\hline 12 & 5 & $21^{* *}$ & 11 & 6 \\
\hline Total & 73 & $204^{* * *}$ & $148^{* *}$ & 104 \\
\hline
\end{tabular}

a Total number of intact linkage groups originating from $F$. circinatum.

${ }^{\mathrm{b}}$ Total number of intact linkage groups originating from $F$. subglutinans.

${ }^{\mathrm{c}}$ Significant deviation for progeny with an intact linkage group from each parent.

Significant deviation is noted as follows: ${ }^{*} 5 \%,{ }^{* *} 1 \%$ and ${ }^{* * *} 0.1 \%$. 
${ }^{\mathrm{d}}$ Total number of framework markers originating from the $F$. circinatum parent.

${ }^{\mathrm{e}}$ Total number of framework markers originating from the $F$. subglutinans parent.

${ }^{\mathrm{f}}$ Significant deviation from the expected 1:1 marker frequency from each parent. Significant deviation is noted as follows: ${ }^{*} 5 \%,{ }^{* *} 1 \%$ and ${ }^{* * *} 0.1 \%$.

\subsection{Bin mapping of accessory markers}

Using MapPop and MAPMAKER, $82 \%$ of the remaining markers were placed in the intervals between framework map markers as well as outside the terminal markers (Table 3). Approximately $10 \%$ of markers were not mapped to the framework maps. This is most likely due to scoring error or the markers being too distant from terminal markers $(\theta>0.45)$ to include them in the final map.

\subsection{Estimated genome coverage and length}

Estimation of genome length using the method of Hulbert ([Hulbert et al., 1988] and [Chakravarti et al., 1991]) showed that the Hulbert estimate was 16\% lower than the observed map length for the $F_{1}$ map (Table 3). Using the Hulbert estimate of genome length, an estimated $99 \%$ of loci in the $\mathrm{F}_{1}$ hybrid map were within $20 \mathrm{cM}$ of a framework marker and an estimated $89 \%$ of loci were within $10 \mathrm{cM}$ of a framework marker (Table 3).

\section{Discussion}

The $\mathrm{F}_{1}$ progeny analysed in this study were the product of an interspecific cross between $F$. circinatum and F. subglutinans. Because these fungi are haploid, analysis of segregation patterns in $\mathrm{F}_{1}$ progeny is similar to that of a backcross population in a diploid organism. No prior cloning or sequence data were required for the AFLP analyses and a large number of markers (average 45 markers produced per primer combination) were generated. Twelve linkage groups were found for the framework maps, which is consistent with the haploid chromosome number of $F$. subglutinans (Xu et al., 1995). The haploid chromosome number for $F$. circinatum is not known.

In this study, 582 polymorphic markers were generated and of these 252 were used to compile an $\mathrm{F}_{1}$ framework linkage map. Two separate framework maps were also 
generated for the parental strains of this interspecific cross in order to evaluate the stability of marker ordering and map distances in the parental (linkage phase) maps and the $F_{1}$ map. The ordering of markers in two different (mutually exclusive) sets allowed us to independently evaluate the possible local effects of specific marker combinations and their associated errors. As could be expected, the parental maps were generally shorter than the $\mathrm{F}_{1}$ map due to lower map coverage. This is shown clearly in Linkage Group $1 \mathrm{H}$, which has no markers originating from $F$. circinatum at the top end of the linkage group. The parental maps were also less inflated due to the presence of fewer markers. It has been shown previously that the addition of markers expands the length of linkage groups (Jurgenson et al., 2002b), which could be due to possible scoring error (Hackett and Broadfoot, 2003). A lower threshold was used in constructing the parental framework maps so that all markers present in the $F_{1}$ map could also be placed in the parental maps. When the same threshold was used for the parental and the $F_{1}$ maps, several markers could not be placed in the parental maps, especially in cases where map intervals became very large due to low map coverage.

The addition of the parental maps in this study has allowed us to compare the parental maps and the $F_{1}$ map. Even though a reasonable comparison could be drawn between them, significant differences do exist. Increasing the number of markers (as is the case with the $F_{1}$ map) provided better map coverage at linkage group terminals as well as increased statistical rigour to the framework linkage map.

Genetic maps have been published for other Fusarium species ([Xu and Leslie, 1996], [Jurgenson et al., 2002a], [Jurgenson et al., 2002b] and [Gale et al., 2005]). The genetic map of $F$. verticillioides had a total length of $1452 \mathrm{cM}$ and a physical distance per unit of recombination of $\sim 32 \mathrm{~kb} / \mathrm{cM}$ (Xu and Leslie, 1996). Addition of AFLP markers to the existing RFLP map increased the map length to $2188 \mathrm{cM}$ and the physical distance per unit of recombination decreased to $\sim 21 \mathrm{~kb} / \mathrm{cM}$ (Jurgenson et al., 2002b). The genetic map of $F$. graminearum had a map length of $1286 \mathrm{cM}$ (Jurgenson et al., 2002a). However, phylogenetic evidence has subsequently shown that this map was based on an interspecific cross between $F$. graminearum and Fusarium asiaticum (O'Donnell et al., 2004). A genetic map of $F$. graminearum had a map length of $1234 \mathrm{cM}$ (Gale et al., 2005). The $F_{1}$ map in this study had a map length of $2774 \mathrm{cM}$ and the physical distance 
per unit of recombination was $\approx 20 \mathrm{~kb} / \mathrm{cM}$. Thus, the $\mathrm{F}_{1}$ map produced in this study is consistent with previous published maps for Fusarium spp.

The observed map length for $F$. subglutinans in this study was only $5.6 \%$ larger than the F. circinatum map. This is despite the fact that there were $42.3 \%$ more framework markers included in the $F$. circinatum map. Conversely, approximately $60 \%$ of the $\mathrm{F}_{1}$ genome was descended from the F. subglutinans parent. This is explained by the average spacing between framework markers being greater for $F$. subglutinans leading to a larger observed map length.

The two parental species, $F$. circinatum and F. subglutinans, shared 44\% AFLP identity. Leslie et al. (2001) noted that, within the Liseola section of Fusarium, strains that share $>65 \%$ band identity represent the same biological species. In contrast, those of different species usually share no more than $40 \%$ band identity, and often significantly less. Although the two parental strains used in this study represent discrete taxa, our results showed a higher level of band identity (44\%) than isolates studied in other mapping studies using AFLP analysis for intraspecific crosses in Fusarium (Jurgenson et al., 2002a). Furthermore, in the map of an interspecific cross between $F$. graminearum and $F$. asiaticum, although not in the Liseola section of Fusarium, 50\% band identity was observed between the two isolates used to construct the genetic map (Jurgenson et al., 2002a). In an interspecies cross between G. fujikuroi (mating population C) and $G$. intermedia (mating population D) band identity was approximately 50\% ([Desjardins et al., 1997] and [Leslie et al., 2004b]). Although in separate mating populations, the authors hypothesized that these two species might be consolidated into a single species. Thus, genetic similarity as determined from the percentage band identity using AFLPs appears to be consistent with relationships inferred from phylogenetic analyses based on differences in DNA sequences. The two parental strains in this study are different species, but are more closely related than other members of the mating populations in the Liseola section of Fusarium, based on AFLP similarity. Mendel's postulate of segregation dictates that during the formation of gametes, the paired unit factors segregate randomly so that each gamete receives one or the other with equal likelihood (Klug and Cummings, 1994). Zamir and Tadmor (1986) attributed transmission ratio distortion to linkage between markers and genetic factor(s) that affect 
the fitness of gametes leading to unbalanced transmission of parental alleles to the next generation. In the present study, there was genome-wide selection for alleles of the $F$. subglutinans parent, with an estimated $59.8 \%$ of $\mathrm{F}_{1}$ progeny genomes being received from the $F$. subglutinans parent. The $\mathrm{F}_{1}$ progeny also showed a tendency to inherit intact parental linkage groups originating from the F. subglutinans parent (Table 4). Of the 292 markers that exhibited transmission ratio distortion, 96\% were skewed towards the $F$. subglutinans parent. This interspecies cross therefore showed a clear bias towards the transmission of $F$. subglutinans alleles. We were not able to determine what proportion of the distorted loci exhibited epistatic interactions. However, $15 \%$ of the distorted markers exhibited a segregation ratio of approximately $3: 1(P<0.05)$. In haploid organisms this is an indication of epistasis with two independent loci being involved in producing the phenotypic trait.

Marker loci exhibiting transmission ratio distortion suggest the presence of a distorting genetic factor in that region of the genome. However, it has also been shown that the greater the genetic divergence between the parental lines, the higher the levels of transmission ratio distortion ([Paterson et al., 1991] and [Grandillo and Tanksley, 1996]). In the present study, approximately $50 \%$ of the markers showed transmission ratio distortion. A more extreme case of transmission ratio distortion was reported in an interspecific cross between the domesticated tomato (Lycopersicon esculentum) and a wild relative (Lycopersicon pennellii), which showed 80\% skewed segregation (DeVicente and Tanksley, 1993). Transmission ratio distortion ranging from 11 to 66\% has also been reported in basidiomycetous fungi (e.g. [Larraya et al., 2000] and [Lind et al., 2005]) as well as in other ascomycetes (e.g. [Xu and Leslie, 1996], [Leslie et al., 2004b] and [Gale et al., 2005]). The authors of these studies have hypothesized that the distortion could be attributed to several factors such as bias in the collection of spores used for the mapping population ([Larraya et al., 2000] and [Lind et al., 2005]), error in scoring (Xu and Leslie, 1996), differential viability of certain ascospores (Xu and Leslie, 1996), or to structural rearrangements of chromosomes that may have caused distorted segregation patterns (Gale et al., 2005).

The $F$. subglutinans $\times F$. circinatum cross showed a clear preferential inheritance of alleles as well as complete chromosomes from the $F$. subglutinans parent rather than 
from the $F$. circinatum genome. This suggests a general fitness benefit for $\mathrm{F}_{1}$ progeny that inherited $F$. subglutinans alleles. The reasons for this unidirectional distortion are not clear at present. It is possible that ascospores with $F$. subglutinans alleles were generally more viable on the specific crossing medium that was used, or that there were fewer negative interactions of $F$. subglutinans alleles with the hybrid genetic background. In this case, fitness selection would be due to the additive effects of individual genetic factors as mentioned previously. It is also possible that selection occurred in some cases against recombinant gametes because of co-evolved gene complexes that were broken up by recombination (Jurgenson et al., 2002a), but this would not explain the unidirectional bias observed.

Despite the use of the biological species concept in species delineation, interspecific crosses in Fusarium section Liseola have been reported previously. Fusarium fujikuroi (mating population C) and Fusarium proliferatum (mating population D) are defined as being different biological species, yet a few isolates have been shown to be sexually compatible ([Desjardins et al., 1997] and [Leslie et al., 2004b]), indicating the limitations of the biological species concept in the Liseola section of Fusarium. A naturally occurring hybrid has also been identified (Leslie et al., 2004a). One hypothesis to explain the presence of a naturally occurring hybrid is that of a hybrid swarm, possibly being geographically separated or occurring on a specific host. The standard tester strains are represented by distinct species, but a hybrid swarm might naturally exist between $F$. fujikuroi and F. proliferatum (Leslie et al., 2004b). The authors hypothesized that these two species might be consolidated into a single species. It may also be possible that the two species are in the final stages of speciation with some individuals in each species still being able to overcome crossing barriers. In the present study, isolates representing mating populations $\mathrm{E}$ and $\mathrm{H}$ of $G$. fujikuroi are phylogenetically closely related ([O’Donnell and Cigelnik, 1997] and [O’Donnell et al., 1998]), but are less similar to each other than mating populations C and D are to each other (Steenkamp et al., 2001). It is also important to highlight the fact that progeny used in this study were obtained from a laboratory cross rather from a natural cross and that only one isolate of $F$. subglutinans has been found to cross to one isolate of $F$. circinatum. The absence of host-specific 
factors in the laboratory cross may have helped to overcome natural crossing barriers between these two species.

The framework map generated in this study will be used to identify QTLs for important quantitative traits such as pathogenicity in $F$. circinatum, which is an economically important pine tree pathogen. In a previous study (Friel et al., 2002), $F_{1}$ progeny of this same cross were found to be avirulent on pine trees. However, a backcross population involving a single $\mathrm{F}_{1}$ individual crossed to the $F$. circinatum parental strain exhibited a wide range of virulence. We have constructed a similar backcross (unpublished results). This experimental population would be useful to identify QTLs associated with pathogenicity in the $F$. circinatum and $F$. subglutinans genomes and will aid us in gaining a better understanding of the genetic basis of $F$. circinatum virulence on Pinus species. It is reasonable to expect that there would be significant synteny between the genomes of $F$. subglutinans and $F$. circinatum, and that of $F$. verticillioides, which is completely sequenced and publicly available (http://www.broad.mit.edu). These resources should facilitate further investigation into the genetic determinants of pathogenicity of the pitch canker fungus.

The framework linkage map generated in this study will provide a means for studying genetic architecture of crossing barriers between $F$. subglutinans and $F$. circinatum. This will be possible with a more in depth analysis of the genome-wide pattern of segregation distortion observed in this mapping study. As mentioned, the genomic sequence of $F$. verticillioides is available and this genome is comparable to that of $F$. subglutinans in that there are also 12 chromosomes and the genome size is similar (Xu and Leslie, 1995). By sequencing selected AFLP framework markers generated in this study and finding a matching sequence in the $F$. verticillioides genome, we should be able to link the 12 linkage groups of this study to the F. verticillioides chromosomes. Putative hybrid fitness loci and pathogenesis-related QTLs could be fine-mapped and possibly identified by studying the corresponding genomic regions in the $F$. verticillioides genome. 


\section{References}

Britz et al., 1999 H. Britz, T.A. Coutinho, M.J. Wingfield, W.F.O. Marasas, T.R. Gordon and J.F. Leslie, Fusarium subglutinans f. sp. pini represents a distinct mating population in the Gibberella fujikuroi species complex, Appl. Environ. Microbiol. 65 (1999), pp. $1198-1201$.

Chakravarti et al., 1991 A. Chakravarti, L.K. Lasher and J.E. Reefer, A maximum likelihood method for estimating genome length using genetic linkage data, Genetics 128 (1991), pp. 175-182.

Desjardins et al., 1997 A.E. Desjardins, R.D. Plattner and R.E. Nelson, Production of fumonisin $\mathrm{B}_{1}$ and moniliformin by Gibberella fujikuroi from rice from various geographic areas, Appl. Environ. Microbiol. 63 (1997), pp. 1838-1842.

Desjardins et al., 2000 A.E. Desjardins, R.D. Plattner and T.R. Gordon, Gibberella fujikuroi mating population A and Fusarium subglutinans from teosinte species and maize from Mexico and Central America, Mycol. Res. 104 (2000), pp. 865-872. DeVicente and Tanksley, 1993 M.C. DeVicente and S.D. Tanksley, QTL analysis of transgressive segregation in an interspecific tomato cross, Genetics 134 (1993), pp. 585596.

Friel et al., 2002 C.J. Friel, S.C. Kirkpatrick and T.R. Gordon, Virulence to pine in the progeny of a hybrid cross in the Gibberella mating population complex, Phytopathology 92 (2002), p. S27.

Gale et al., 2005 L.R. Gale, J.D. Bryant, S. Calvo, H. Giese, T. Katan, K. O’Donnell, H. Suga, M. Taga, T.R. Usgaard, T.J. Ward and H.C. Kistler, Chromosome complement of the fungal plant pathogen Fusarium graminearum based on genetic and physical mapping and cytological observations, Genetics 171 (2005), pp. 985-1001.

Grandillo and Tanksley, 1996 S. Grandillo and S.D. Tanksley, Genetic analysis of RFLPs, GATA microsatellites and RAPDs in a cross between L. esculentum and $L$. pimpenillifolium, Theor. Appl. Genet. 92 (1996), pp. 957-965.

Hackett and Broadfoot, 2003 C.A. Hackett and L.B. Broadfoot, Effects of genotyping errors, missing values and segregation distortion in molecular marker data on the construction of linkage maps, Heredity 90 (2003), pp. 33-38. 
Hepting and Roth, 1946 G.H. Hepting and E.R. Roth, Pitch canker, a new disease of some southern pines, J. For. 44 (1946), pp. 742-744.

Hulbert et al., 1988 S.H. Hulbert, T.W. Ilott, E.J. Legg, S.E. Lincoln, E.S. Lander and R.W. Michelmore, Genetic analysis of the fungus, Bremia lactucae, using restriction fragment length polymorphisms, Genetics 120 (1988), pp. 947-958.

Jurgenson et al., 2002a J.E. Jurgenson, R.L. Bowden, K.A. Zeller, J.F. Leslie, N.J. Alexander and R.D. Plattner, A genetic map of Gibberella zeae (Fusarium graminearum), Genetics 160 (2002), pp. 1451-1460.

Jurgenson et al., 2002b J.E. Jurgenson, K.A. Zeller and J.F. Leslie, Expanded genetic map of Gibberella moniliforme (Fusarium verticillioides), Appl. Environ. Microbiol. 68 (2002), pp. 1972-1979.

Klug and Cummings, 1994 W.S. Klug and M.R. Cummings, Concepts of Genetics, Prentice-Hall, Inc, New Jersey, USA (1994).

Lander et al., 1987 E.S. Lander, P. Green, J. Abrahamson, A. Barlow, M.J. Daly, S.E. Lincoln and L. Newburg, MAPMAKER: an interactive computer package for constructing primary genetic linkage maps of experimental and natural populations, Genomics 1 (1987), pp. 174-181.

Lange and Boehnke, 1982 K. Lange and M. Boehnke, How many polymorphic genes will it take to span the human genome?, Am. J. Hum. Genet. 34 (1982), pp. 842-845.

Larraya et al., 2000 L.M. Larraya, G. Pérez, E. Ritter, A.G. Pisabarro and L. Ramírez, Genetic linkage map of the edible basidiomycete Pleurotus ostreatus, Appl. Environ. Microbiol. 66 (2000), pp. 5290-5300.

Lepoint et al., 2005 P.C.E. Lepoint, F.T.J. Munaut and H.M.M. Maraite, Gibberella xylarioides sensu lato from Coffea canephora: A new mating population in the Gibberella fujikuroi species complex, Appl. Environ. Microbiol. 71 (2005), pp. 84668471.

Leslie et al., 2001 J.F. Leslie, K.A. Zeller and B.A. Summerell, Icebergs and species in populations of Fusarium, Physiol. Mol. Plant Pathol. 59 (2001), pp. 107-117.

Leslie et al., 2004a J.F. Leslie, K.A. Zeller, A. Logrieco, G. Mulè, A. Moretti and A. Ritieni, Species diversity of and toxin production by Gibberella fujikuroi species 
complex strains isolated from native prairie grasses in Kansas, Appl. Environ. Microbiol. 70 (2004), pp. 2254-2262.

Leslie et al., 2004b J.F. Leslie, K.A. Zeller, M. Wohler and B.A. Summerell, Interfertility of two mating populations in the Gibberella fujikuroi species complex, Eur. J. Plant Path. 110 (2004), pp. 611-618.

Lind et al., 2005 M. Lind, Å. Olson and J. Stenlid, An AFLP-markers based genetic linkage map of Heterobasidion annosum locating intersterility genes, Fung. Genet. Biol. 42 (2005), pp. 519-527.

McCain et al., 1987 A.H. McCain, C.S. Koehler and S.A. Tjosvold, Pitch canker threatens California pines, Calif. Agric. 41 (1987), pp. 22-23. Myburg et al., 2001 A.A. Myburg, D.L. Remington, D.M. O’Malley, R.R. Sederoff and R.W. Whetten, High-throughput AFLP analysis using infrared dye-labeled primers and an automated DNA sequencer, BioTechniques 30 (2001), pp. 348-357. Myburg et al., 2003 A.A. Myburg, A.R. Griffin, R.R. Sederoff and R.W. Whetten, Comparative genetic linkage maps of Eucalyptus grandis, Eucalyptus globulus and their $\mathrm{F}_{1}$ hybrid based on a double pseudo-backcross mapping approach, Theor. Appl. Genet. 107 (2003), pp. 1028-1042.

Nirenberg and O’Donnell, 1998 H.I. Nirenberg and K. O’Donnell, New Fusarium species and combinations within the Gibberella fujikuroi species complex, Mycologia 90 (1998), pp. $434-458$.

O’Donnell and Cigelnik, 1997 K. O’Donnell and E. Cigelnik, Two divergent intragenomic rDNA ITS2 types within a monophyletic lineage of the fungus Fusarium are nonorthologous, Mol. Phyl. Evol. 7 (1997), pp. 103-116.

O’Donnell et al., 1998 K. O’Donnell, E. Cigelnik and H.I. Nirenberg, Molecular systematics and phylogeography of the Gibberella fujikuroi species complex, Mycologia 90 (1998), pp. 465-493.

O’Donnell et al., 2004 K. O’Donnell, T.J. Ward, D.M. Geiser, H.C. Kistler and T. Aoki, Genealogical concordance between the mating type locus and seven other nuclear genes supports formal recognition of nine phylogenetically distinct species within the Fusarium graminearum clade, Fung. Gen. Biol. 41 (2004), pp. 600-623. 
Paterson et al., 1991 A.H. Paterson, S. Damon, J.D. Hewitt, D. Zamir, H.D. Rabinowitch, S.E. Lincoln, E.S. Lander and S.D. Tanksley, Mendelian factors underlying quantitative traits in tomato: Comparison across species, generations, and environments, Genetics 127 (1991), pp. 181-197.

Phan et al., 2004 H.T. Phan, L.W. Burgess, B.A. Summerell, S. Bullock, E.C.Y. Liew, J.L. Smith-White and J.R. Clarkson, Gibberella gaditjirrii (Fusarium gaditjirrii) sp. nov., a new species from tropical grasses in Australia, Stud. Mycol. 50 (2004), pp. 261-272. Raeder and Broda, 1985 U. Raeder and P. Broda, Rapid preparation of DNA from filamentous fungi, Let. Appl. Microbiol. 1 (1985), pp. 17-20.

Sambrook et al., 1989 J. Sambrook, E.F. Fritsch and T. Maniatis, Molecular cloning: A Laboratory Manual, Cold Spring Harbor Laboratory Press, New York (1989) pp. E.3E.4.

Samuels et al., 2001 G.J. Samuels, H.I. Nirenberg and K.A. Seifert, Perithecial species of Fusarium. In: B.A. Summerell, J.F. Leslie, D. Backhouse, W.L. Bryden and L.W. Burgess, Editors, Fusarium: Paul E. Nelson Memorial Symposium, APS Press, Minnesota (2001), pp. 1-14.

Steenkamp et al., 1999 E.T. Steenkamp, B.D. Wingfield, T.A. Coutinho, M.J. Wingfield and W.F.O. Marasas, Differentiation of Fusarium subglutinans f. sp. pini by histone gene sequence data, Appl. Environ. Microbiol. 65 (1999), pp. 3401-3406.

Steenkamp et al., 2000 E.T. Steenkamp, B.D. Wingfield, T.A. Coutinho, K.A. Zeller, M.J. Wingfield, W.F.O. Marasas and J.F. Leslie, PCR-based identification of MAT-1 and MAT-2 in the Gibberella fujikuroi species complex, Appl. Environ. Microbiol. 66 (2000), pp. 4378-4382.

Steenkamp et al., 2001 E.T. Steenkamp, T.A. Coutinho, A.E. Desjardins, B.D. Wingfield, W.F.O. Marasas and M.J. Wingfield, Gibberella fujikuroi mating population E is associated with maize and teosinte, Mol. Plant Pathol. 2 (2001), pp. 215-221.

Steenkamp et al., 2002 E.T. Steenkamp, B.D. Wingfield, A.E. Desjardins, W.F.O. Marasas and M.J. Wingfield, Cryptic speciation in Fusarium subglutinans, Mycologia 94 (2002), pp. 1032-1043.

Van Berloo, 1999 R. Van Berloo, GGT: software for the display of graphical genotypes, J. Hered. 90 (1999), pp. 328-329. 
Viljoen and Wingfield, 1994 A. Viljoen and M.J. Wingfield, First report of Fusarium subglutinans f. sp. pini on pine seedlings in South Africa, Plant Dis. 78 (1994), pp. 309312.

Vision et al., 2000 T.J. Vision, D.G. Brown, D.B. Shmoys, R.T. Durrett and S.D. Tanksley, Selective mapping: a strategy for optimizing the construction of high-density linkage maps, Genetics 155 (2000), pp. 407-420.

Vos et al., 1995 P. Vos, R. Hogers, M. Bleeker, M. Reijans, T. Van der Lee, M. Hornes, A. Frijters, J. Pot, J. Peleman, M. Kuiper and M. Zabeau, AFLP: a new technique for DNA fingerprinting, Nucl. Acids Res. 23 (1995), pp. 4407-4414.

Xu et al., 1995 J.-R. Xu, K. Yan, M.B. Dickman and J.F. Leslie, Electrophoretic karyotypes distinguish the biological species of Gibberella fujikuroi (Fusarium section Liseola), Mol. Plant-Microbe Inter. 8 (1995), pp. 74-84.

$\mathrm{Xu}$ and Leslie, 1996 J.-R. Xu and J.F. Leslie, A genetic map of Gibberella fujikuroi mating population A (Fusarium moniliforme), Genetics 143 (1996), pp. 175-189. Zamir and Tadmor, 1986 D. Zamir and Y. Tadmor, Unequal segregation of nuclear genes in plants, Bot. Gaz. 147 (1986), pp. 355-358. Full Text via CrossRef Zeller et al., 2000 K.A. Zeller, J.E. Jurgenson, E.M. El-Assiuty and J.F. Leslie, Isozyme and amplified fragment length polymorphisms from Cephalosporium maydis in Egypt, Phytoparasitica 28 (2000), pp. 121-130.

Zeller et al., 2003 K.A. Zeller, B.A. Summerell, S. Bullock and J.F. Leslie, Gibberella fujikuroi (Fusarium konzum) sp. nov. from prairie grasses, a new species in the Gibberella fujikuroi species complex, Mycologia 95 (2003), pp. 943-954.

Corresponding author. Present address: Department of Genetics, Faculty of Natural and Agricultural Sciences, University of Pretoria, Pretoria 0001, South Africa. Fax: +27 12 4203947. 